Research Paper

\title{
Neutrophil Granulocytes in Ovarian Cancer - Induction of Epithelial-To-Mesenchymal-Transition and Tumor Cell Migration
}

\author{
Christine Mayer ${ }^{1}$, Silvia Darb-Esfahani2,3, Anne-Sophie Meyer ${ }^{4}$, Katrin Hübner ${ }^{5}$, Joachim Rom¹, Christof
} Sohn¹, Ioana Braicu ${ }^{3,6}$, Jalid Sehouli3,6, G. Maria Hänsch ${ }^{7}$, Matthias M. Gaida ${ }^{\natural}$

1. Department of Gynecology and Obstetrics, University Hospital Heidelberg, Germany;

2. Institute of Pathology, Charité, University Hospital Berlin, Germany;

3. Tumorbank Ovarian Cancer Network (TOC), Berlin, Germany;

4. Institute of Pathology, University Hospital Heidelberg, Germany;

5. BioQuant, COS Heidelberg, University Heidelberg, Germany;

6. Department of Gynecology, Charité University Hospital Berlin, Germany;

7. Institute of Immunology, University Hospital Heidelberg, Germany.

$\triangle$ Corresponding author: Christine Mayer, M.D. Department of Gynecology and Obstetrics, University Hospital Heidelberg, Im Neuenheimer Feld 440, 69120 Heidelberg, Germany. Telephone: +49-6221-56-7856 Fax:+ 49-6221-56-5233 Email: Christine.Mayer@med.uni-heidelberg.de.

(C) Ivyspring International Publisher. Reproduction is permitted for personal, noncommercial use, provided that the article is in whole, unmodified, and properly cited. See http://ivyspring.com/terms for terms and conditions.

Received: 2015.10.19; Accepted: 2016.01.22; Published: 2016.03.10

\begin{abstract}
Background: Ovarian cancer $(\mathrm{OvCa})$ is a highly aggressive malignoma with a tumor-promoting microenvironment. Infiltration of polymorphonuclear neutrophils (PMN) is frequently seen, raising the question of their impact on tumor development. In that context, effects of PMN on human ovarian cancer cells were assessed.

Methods: Human epithelial ovarian cancer cells were incubated with human PMN, lysate of PMN, or neutrophil elastase. Morphological alterations were observed by time-lapse video-microscopy, and the underlying molecular mechanism was analyzed by flow cytometry and Western blotting. Functional alternations were assessed by an in vitro wound healing assay. In parallel, a large cohort of $n=334$ primary OvCa tissue samples of various histological subtypes was histologically evaluated.

Results: Co-cultivation of cancer cells with either PMN or PMN lysate causes a change of the polygonal epithelial phenotype of the cells towards a spindle shaped morphology, causing a cribriform cell growth. The PMN-induced alteration could be attributed to elastase, a major protease of PMN. Elastase-induced shape change was most likely due to the degradation of membranous E-cadherin, which results in loss of cell contacts and polarity. Moreover, in response to elastase, epithelial cytokeratins were downmodulated, in parallel with a nuclear translocation of $\beta$-catenin. These PMN-elastase induced alterations of cells are compatible with an epithelial-to-mesenchymal transition (EMT) of the cancer cells. Following EMT, the cells displayed a more migratory phenotype. In human biopsies, neutrophil infiltration was seen in $72 \%$ of the cases. PMN infiltrates were detected preferentially in areas with low E-cadherin expression.
\end{abstract}

Conclusion: PMN in the microenvironment of OvCa can alter tumor cells towards a mesenchymal and migratory phenotype.

Key words: Ovarian cancer, neutrophils, neutrophil elastase, tumor microenvironment, epithelial to mesenchymal transition, migration.

\section{Introduction}

Ovarian cancer (OvCa) is a highly aggressive malignoma ranking as the fifth highest cause of cancer-related mortality in women in the USA [1].
Symptoms of the disease usually develop at a very late stage; therefore, about $70 \%$ of patients are already in an advanced stage of the disease at the time of di- 
agnosis (FIGO III or IV). Surgical tumor removal is the primary treatment in combination with chemotherapy $[2,3]$. Despite improved surgical procedures and a high primary response to chemotherapy, about $70 \%$ of patients with advanced ovarian cancer develop a tumor relapse and die from the disease [1].

To develop new strategies for further therapeutic interventions, a detailed understanding of the molecular mechanisms in ovarian cancer is necessary. In the recent years, it became apparent that the microenvironment greatly affects the development of the tumor because it is a major source of tumor promoting factors in a huge variety of tumors, including OvCa. Particularly tumor infiltrating leukocytes can alter tumorcells towards an aggressive phenotype [4,5]. In this context, the role of polymorphonuclear neutrophils (PMN) in ovarian cancer is of great interest. Early data suggested that activated PMN have the ability to lyse ovarian cancer cells [6]. However, more recent data indicate that a shift of the PMN to T cells ratio towards PMN is associated with more aggressive disease in OvCa $[7,8]$. Furthermore, it was shown that peripheral neutrophils of ovarian cancer patients displayed a "proinflammatory phenotype" characterized by an increased production of reactive oxygen species and enhanced expression of adhesion molecules, when compared to healthy blood donors. A neutrophil-tumor cell contact mediated this phenotype changes of PMN [9]. For other (gynecological) malignomas, such as breast cancers, an increased metastatic potential has been described correlating with increased levels of PMN [10,11]. One hypothesis is that PMN-derived matrix-degrading proteases are responsible for these changes [12]. To attain metastatic ability, carcinoma cells display phenotypic changes, particularly an epithelial to mesenchymal transition (EMT). EMT is characterized by the loss of the intercellular adhesion molecules E-cadherin, downmodulation of cytokeratins, and a transfer of $\mathrm{B}$-catenin from the cytoplasm to the nucleus $[13,14]$. Several studies, for example in breast cancer, showed a correlation between the loss of E-cadherin and an increased tumor progression, the development of distant metastasis and recurrence of the disease $[15,16]$.

Since the role of PMN in ovarian cancer, especially with regard to tumor cell migration and aggressiveness, is not yet understood, co-culture experiments of ovarian cancer cells and human PMN were performed. PMN, though primarily studied in the context of host defense, participate in acute and chronic inflammatory disease and most likely also in tissue turnover, due to the production of short-lived reactive oxygen species, and their diverse and large reservoir of proinflammatory cytokines, and tissue degrading enzymes, including serine -and metallo- proteases. We now detected a dyshesion of tumor cells when in contact with PMN, and more specifically with PMN-derived elastase. Dyshesion was due to the enzymatic digestion of the intercellular adhesion molecule E-cadherin and resulted in the transition to a mesenchymal and migratory phenotype. In a large series of human ovarian cancer samples, we could detect PMN as a significant portion of tumor infiltrating immune cells and found an association with the expression of the EMT transcription factor ZEB1 which was also often present in areas where a loss of tumor-expressed E-cadherin occurred. Our results indicate that PMN are able to alter the ovarian cancer towards an aggressive "mesenchymal" phenotype.

\section{Material and methods}

\section{Tissue samples}

Tissue samples of 334 patients with primary ovarian carcinoma who underwent radical surgery with the aim of total cytoreduction were investigated. Resection specimens were formalin-fixed and paraffin-embedded (FFPE) for diagnostic purposes at the Institute of Pathology of the Charité Universitätsmedizin Berlin. 238 patients had been included into the TOC project (Tumorbank Ovarian Cancer, www.toc-network.de). Scientific use of TOC and non-TOC cases has been approved by the ethics committee of the Charité. Hematoxylin-eosin-stained slides were re-evaluated according to histology by an experienced gynecopathologist (SDE). Typing as high-grade serous was performed by current WHO criteria. Most cases were high-grade serous carcinomas $(n=213,63.8 \%)$, further histological subtypes were low-grade serous carcinomas $(n=46,13.8 \%)$, (low-grade) endometrioid ( $\mathrm{n}=34,10.2 \%)$, and clear cell carcinomas $(n=41,12.3 \%)$. The distribution of clinico-pathological parameters in the study cohort is shown in table 1 .

\section{Immunohistochemistry}

Tissue microarrays with a core size of $1 \mathrm{~mm}$ respectively $1.5 \mathrm{~mm}$ in diameter (two cores per sample) were performed of FFPE resection specimens. Then standard hematoxylin/eosin (H\&E) staining as well as immunohistochemistry was performed. The following primary antibodies were used: anti-CD15 (DAKO, IS062, 1:50, retrieval condition: $\mathrm{pH}$ 6.0), anti-E-cadherin (DAKO, NCH-38, 1:10, retrieval condition: pH 9.0), ZEB1/AREB6 (abcam, 416A7H10, 1:200, retrieval condition: $\mathrm{pH}$ 6.0) As secondary antibody either the Histofine, Simple Stain universal polymer was used (Nichirei, Tokyo, Japan), followed by the color reaction using liquid permanent red (Zytomed, Berlin, Germany), or the Dako EnVision kit (Dako) was used, both followed by counter stain with hema- 
toxylin. The amount of E-cadherin expression was determined using the Allred score (score $0-8)$, calculated by the sum of staining intensity $(0=$ no; $1=$ weak; $2=$ moderate; $3=$ strong) and distribution $(0=$ $0 \% ; 1=<1 \% ; 2=1-10 \% ; 3=>10-33 \%, 4=>33-66 \%, 5=$ $>66-100 \%$ positive cells). The number of tumor infiltrating neutrophils was judged by a semiquantitative scoring system (negative: score $0=0$, moderate: score $1=1-19$, high: score $2=\geq 20 \mathrm{PMN} / 1 \mathrm{~mm}$ diameter core) and the nuclear translocation of ZEB1 was judged present or absent $(0=$ absent; $1=$ present $)$.

Table 1. Clinico-pathological parameters: Tissue samples of 334 patients with primary ovarian carcinoma who underwent radical surgery with the aim of total cytoreduction were investigated and the distribution of clinico-pathological parameters in the study cohort is shown.

\begin{tabular}{|c|c|c|c|c|}
\hline & $\begin{array}{c}\text { high-grade } \\
\text { serous }(n=213) \\
n(\%)\end{array}$ & $\begin{array}{l}\text { low-grade } \\
\text { serous } \\
(n=46) n(\%)\end{array}$ & $\begin{array}{l}\text { endometrioid } \\
\text { (low-grade) } \\
(\mathbf{n}=34) \mathrm{n}(\%)\end{array}$ & $\begin{array}{l}\text { clear cell } \\
(n=41) n(\%)\end{array}$ \\
\hline \multicolumn{5}{|l|}{ E-Cadherin } \\
\hline score $0-1$ & $58(27.9)$ & $16(47.1)$ & $8(23.5)$ & $5(12.5)$ \\
\hline score $2-5$ & $110(52.8)$ & $11(32.4)$ & $17(50.0)$ & $15(37.5)$ \\
\hline score $6-8$ & 40 (19.2) & $9(26.4)$ & $9(26.5)$ & $20(50.0)$ \\
\hline missing & 5 & & & 1 \\
\hline \multicolumn{5}{|l|}{ ZEB1 nuclear } \\
\hline negative & $135(63.4)$ & $15(44.1)$ & $24(70.6)$ & $26(63.4)$ \\
\hline positive & $78(36.6)$ & 19 (55.9) & $10(29.4)$ & $15(36.6)$ \\
\hline \multicolumn{5}{|l|}{ CD15 } \\
\hline $0 /$ core & $53(24.9)$ & $12(36.4)$ & $7(20.6)$ & $15(38.5)$ \\
\hline 1-19/core & $123(57.7)$ & $14(42.4)$ & $20(58.8)$ & $17(43.6)$ \\
\hline$>=20 /$ core & 37 (17.4) & $7(21.2)$ & $7(20.6)$ & 7 (17.9) \\
\hline \multicolumn{5}{|l|}{ age } \\
\hline$<=60$ years & $108(50.7)$ & $18(52.9)$ & $18(52.9)$ & $17(41.5)$ \\
\hline$>60$ years & 105 (49.3) & $16(47.1)$ & $16(47.1)$ & $24(58.5)$ \\
\hline \multicolumn{5}{|l|}{ FIGO stage } \\
\hline FIGO I & $16(7.5)$ & $3(9.1)$ & $16(50.0)$ & $24(58.5)$ \\
\hline FIGO II & $13(6.1)$ & $2(6.1)$ & $4(12.5)$ & $2(4.9)$ \\
\hline FIGO III & $160(75.1)$ & $27(81.8)$ & $12(37.5)$ & $15(36.6)$ \\
\hline FIGO IV & $24(11.3)$ & $1(3.0)$ & $0(0)$ & $0(0.0)$ \\
\hline missing & & 1 & 2 & \\
\hline \multicolumn{5}{|l|}{ residual tumor } \\
\hline none & $98(65.3)$ & $23(88.5)$ & $8(100.0)$ & $9(69.2)$ \\
\hline any & $52(34.7)$ & $3(11.5)$ & $0(0.0)$ & $4(30.8)$ \\
\hline n.d. (FIGO I) & 16 & 2 & 16 & 24 \\
\hline missing & 47 & 6 & 10 & 4 \\
\hline \multicolumn{5}{|l|}{ chemotherapy } \\
\hline platinum-based & $160(75.5)$ & $26(76.5)$ & $16(94.1)$ & $24(88.0)$ \\
\hline other & $5(2.4)$ & $0(0)$ & $0(0.0)$ & $3(10.0)$ \\
\hline none & $2(0.9)$ & 0 & $1(5.9)$ & $3(10.0)$ \\
\hline missing & 46 & 8 & 17 & 11 \\
\hline
\end{tabular}

\section{Ovarian cancer cell lines}

SKOV-3 cells were cultivated in McCoys medium (Biochrom AG, Berlin, Germany) containing 10\% fetal bovine serum and $1 \%$ penicillin-streptomycin (Invitrogen, Karlsruhe, Germany) at $37^{\circ} \mathrm{C}$ in a $5 \% \mathrm{CO}_{2}$ humidified atmosphere. OVCAR-3 cells purchased from ATCC (Manassas, Virginia, USA) were cultivated in RPMI medium (Invitrogen) containing 10\% fetal bovine serum, $1 \%$ penicillin-streptomycin and
$0.01 \mathrm{mg} / \mathrm{ml}$ insulin under conditions described above.

\section{Isolation of PMN and preparation of PMN ly- sate}

Blood from healthy human donors was extracted from peripheral veins and collected in heparin-coated syringes (Informed consent was obtained from the donors and the procedure was approved by the local ethics committee). PMN were isolated by centrifugation on PolymorphPrep (Axis-Shield PoC AS, Oslo, Norway) according to the protocol provided by the manufacturer. Isolated PMN were suspended in McCoys medium (Biochrom) and used within one hour. PMN lysate was prepared by the nitrogen cavitation method. PMN in a concentration of $1 \times 10^{8}$ were suspended in $1 \mathrm{ml}$ phosphate buffer and then cavitated in nitrogen for $20 \mathrm{~min}$ at $380 \mathrm{psi}$.

\section{Co-culture of SKOV-3 with PMN, PMN lysate, or PMN elastase}

SKOV-3 $\left(1 \times 10^{5}\right)$ were plated onLab-TEK chambered slides (Nalge Nunc International, Naperville, Illinois, USA) over night for $20 \mathrm{~h}$. The following day,

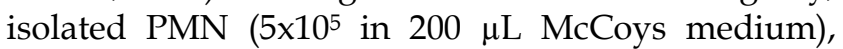
PMN-lysate $(20 \mu \mathrm{L})$, or $3 \mu \mathrm{g}$ PMN-elastase (Calbiochem/Merck, Darmstadt, Germany) were added, and the morphologic changes of SKOV-3 were recorded by time-lapse video microscopy using differential interference contrast (DIC). For the time-lapse-video experiments, PMN were loaded with Calcein AM (1 $\mu \mathrm{g} / \mathrm{ml}$; Sigma Aldrich, Taufkirchen, Germany) for 30 min at $37^{\circ} \mathrm{C}$. MDIC and green fluorescence (calcein) images were taken every 60 seconds for up to 6 hours with a Nikon Ti inverted microscope equipped with an Hamamatsu 1394 ORCA-ERA camera, S Fluor 20x NA 0.75 objective and a perfect focus system (all Nikon, Tokyo, Japan). Images were analyzed with Image $\mathrm{J}$ (NIH, Bethesda, MD, USA).

\section{Protein isolation, SDS-Page, and Western Blot}

Proteins from $5 \times 10^{6}$ SKOV-3 cells were isolated using $150 \mu \mathrm{L}$ RIPA lysis buffer including protease and phosphatase inhibitors (Santa Cruz, Dallas, Texas, USA) and the whole lysate was used for Western blot analysis of cytokeratin and alpha-smooth muscle actin (a-SMA) using a mouse monoclonal antibody to cytokeratin (Santa Cruz, $1 \mu \mathrm{g} / \mathrm{mL}$ ), or mouse monoclonal antibody to a-SMA (Santa Cruz, $1 \mu \mathrm{g} / \mathrm{mL}$ ). For detection of E-cadherin degradation and translocation of $\beta$-catenin to the nucleus the cell was fractionated using the ProteoExtract subcellular proteome extraction kit (Calbiochem/Merck). With this kit the cytosolic fraction, membrane/organelle protein fraction, nucleic protein fraction, and cytoskeletal fraction can be separated. A rabbit polyclonal antibody to 
E-cadherin was obtained from Santa Cruz and used in a final dilution of 1:2000. The antibody to $\beta$-Catenin was purchased from Becton and Dickinson and used in a concentration of $1.25 \mu \mathrm{g} / \mathrm{mL}$. A non-interfering protein assay kit (Calbiochem/Merck) was used for determination of protein concentration in the respective fraction. Lysates or fractions thereof were separated by SDS-PAGE (9\% gel), and the proteins were transferred to nitrocellulose membranes (Whatman, Dassel, Germany). The membranes were then incubated with $5 \%$ nonfat milk for $1 \mathrm{~h}$ at room temperature followed by washing with TRIS-buffered saline-Tween-20 (TBST). The membranes were incubated with the respective antibodies at $4^{\circ} \mathrm{C}$ overnight, bound antibody was detected using POX conjugated secondary antibodies (all from Jackson Immuno Research, USA) and Amersham ECL Plus Western Blotting Detection system (GE Healthcare, Munich, Germany). As loading controls, $\beta$-actin was used, and p84 for the nuclear fraction (antibodies: $\mathrm{Rb} \mathrm{pAb}$ to $\beta$-actin, Abcam, 1:10000; Ms mAb to nuclear matrix protein p84, Abcam $0.1 \mu \mathrm{g} / \mathrm{mL}$ ).

\section{Flow cytometry}

SKOV-3 cells were harvested with a cell scraper, suspended in FACS buffer (PBS containing 1\% bovine serum albumin and $0.1 \%$ sodium acid) and incubated with anti-human E-cadherin (generated in rabbit; Santa Cruz), and anti-rabbit-IgG FITC was used as secondary antibody. Cells were subjected to flow cytometry (BD LSR II) and analysed with cell quest PRO software. $1 \times 10^{4}$ cells were counted and results are expressed as mean fluorescence intensity.

\section{In vitro wound-healing assay}

SKOV-3 cells $\left(8 \times 10^{5} / \mathrm{mL}\right)$ were plated into 6 well plates and grown overnight to near confluence. Cells were either treated with neutrophil elastase (2 $\mu \mathrm{g} / \mathrm{mL}$ ) or left untreated. Using a pipette tip a cell free area was scraped in each well and culture was continued. Images were taken at $0 \mathrm{~h}, 4 \mathrm{~h}$ and $24 \mathrm{~h}$, and cells migrating into the cell free space were quantified.

\section{Statistical analysis}

Statistical analysis was performed using Graph Pad prism software (GraphPad Inc., LaJolla, CA, USA) and the appropriate test. Significance levels were determined at $\mathrm{p}<0.05$.

\section{Results}

\section{Neutrophil infiltration and epitheli- al-to-mesenchymal transition in patients with epithelial ovarian carcinoma}

Biopsies of 319 patients with ovarian carcinoma were analysed. In 232 (72\%), infiltrating PMN were detected as identified by their morphology, expression of CD15, and of elastase (examples in Fig.1). In 58 cases, 20 or more PMN were detected per core, and 2-19 PMN in 174 cases, and 87 cases were considered as negative for PMN (0-1 PMN).PMN infiltration (>1 $\mathrm{PMN} /$ tumor) was detected in all histological subtypes and varied between $61.5 \%$ and $79.5 \%$ with no significant differences between histologic subtypes ( $p=0.157$, chi square test).

The adhesion molecule E-cadherin is strongly expressed on epithelial cells, and in 78 cases a strong and ubiquitous E-cadherin expression was seen (Allred scores 6-8), whereas 153 showed only weak or intermediate staining, (Allred scores 2-5), and 85 cases revealed no staining. E-cadherin expression did not correlate with the numbers of PMN, but correlated significantly with histological subtype: median E-Cadherin Allred scores were 3 in high-grade serous, 4 in low-grade serous, 5 in endometrioid, and 6 in clear cell carcinomas ( $p=0.003$, Mann-Whitney test).

As marker for epithelial-to-mesenchymal transition, expression of ZEB1 in cancer cells was assessed. Nuclear expression of ZEB1 was seen in 122 samples $(37.9 \%)$, with positivity rates varying between $29.4 \%$ and $55.9 \%$. Nuclear expression of ZEB1 did not differ between the histologic subtypes $(\mathrm{p}=0.117$, Chi square test), and expression of ZEB1 did not correlate with the number of infiltrated PMN. There were, however, areas within the biopsies that showed a definite clustering of PMN and ZEB1 positive cancer cells (example in Fig.1).

\section{Neutrophils and neutrophil-derived elastase cause dyshesion of ovarian cancer cell layers}

The clustering of PMN and ZEB1-positive cells prompted a series of experiments on a possible relationship between PMN and EMT. To this end, effects of PMN on ovarian cancer cell lines were assessed. Cells grown to confluence in chambered slides were co-cultured with isolated PMN having incorporated Calcein-AM, and observed by time-lapse video microscopy. A progressive detachment and dispersion of the tumor cells was seen, accompanied by a drastic shape change, but no apparent cell death (Data for the cell line SKOV-3 are shown in Fig.2; images were extracted from the video after 0 h, 1 h, 3 h, and 6 h). Essentially similar data were obtained with another cell line, OVCAR3 (data not shown). Lysates of PMN also caused dyshesion of cancer cells, suggesting a role for PMN-derived proteases. A likely candidate is elastase, and indeed purified elastase induced dyshesion of tumor cells, accompanied by a shape change from a round to ovoid phenotype to an elongated and spindle-shaped appearance, similar to the effects seen with PMN-lysates. 

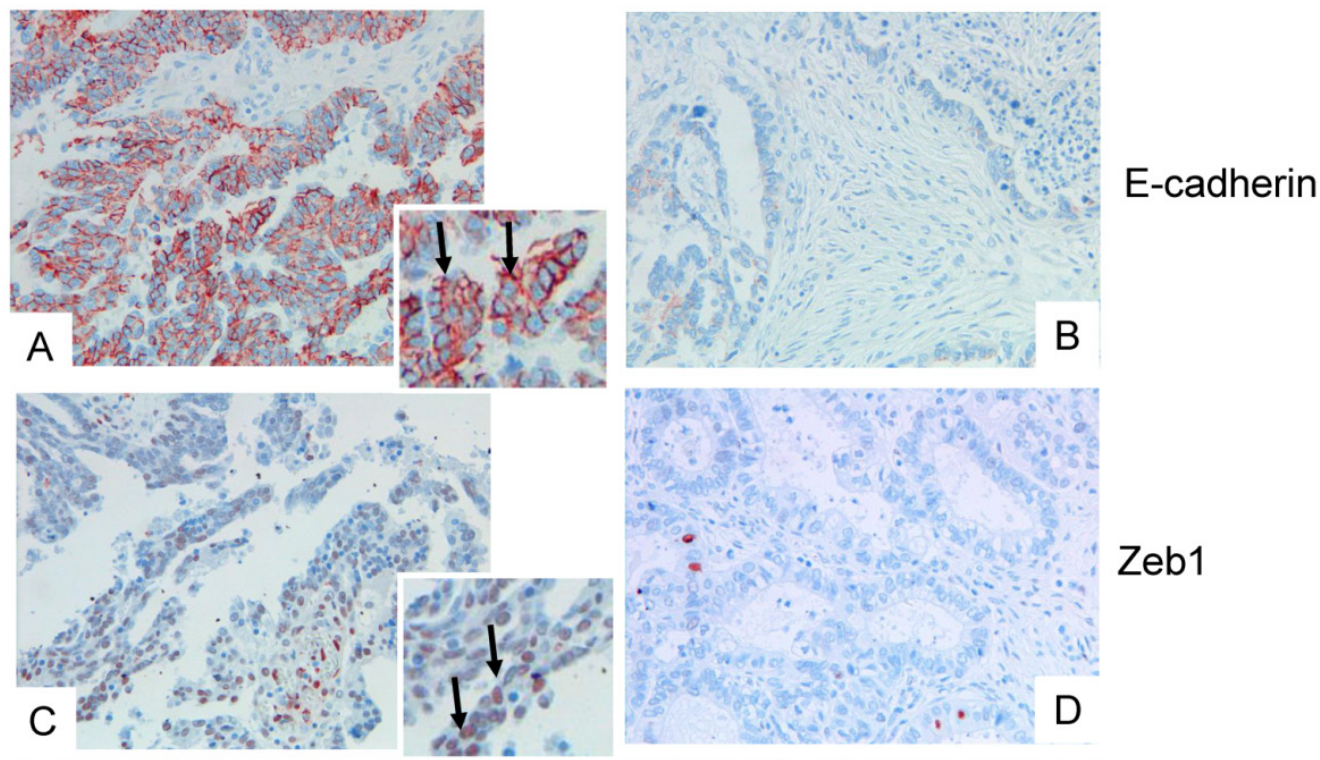

Zeb1
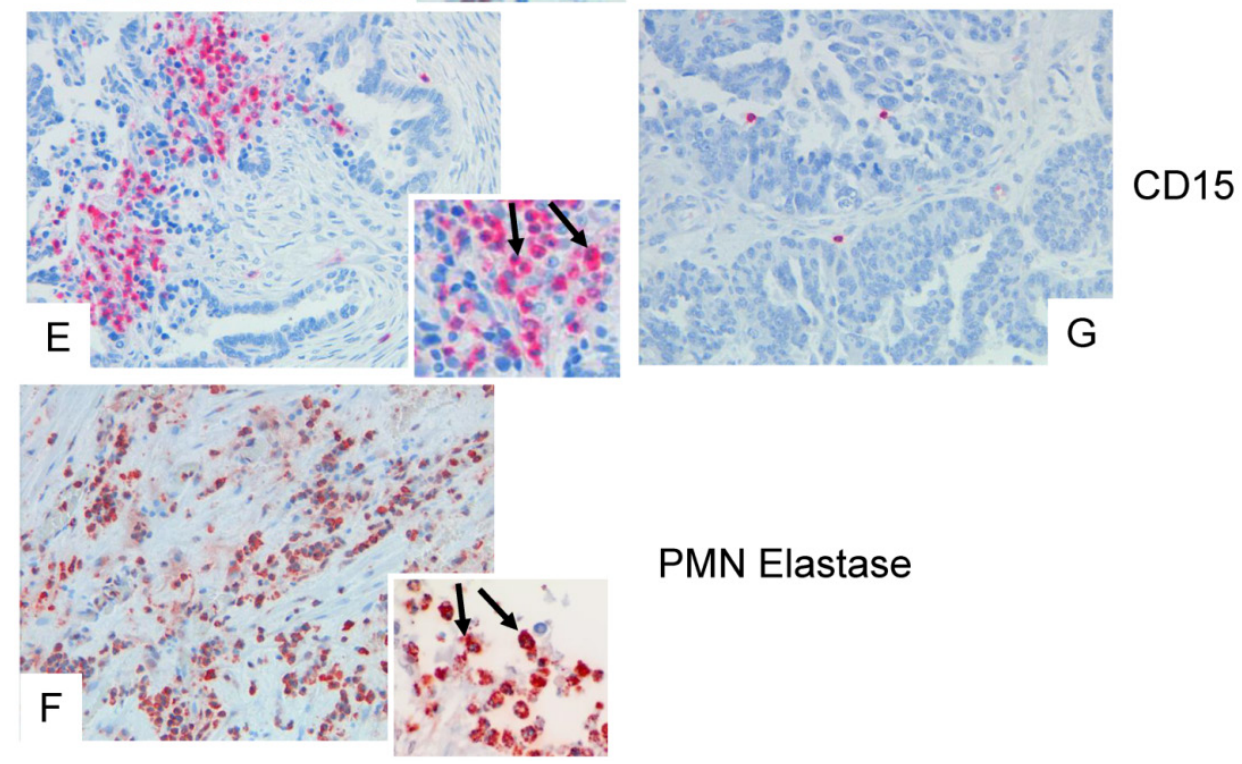

PMN Elastase

Figure 1. Examples of tissue specimens derived from patients with epithelial ovarian cancer: On the left panel, tissue with dense expression of PMN, on the right panel with low expression of PMN are shown (A, B), and corresponding staining for E-cadherin (C, D), and ZEBI (E, F). The inserts show a digital zoom of selected areas, and the arrows show typical examples. In (G) a dense infiltration of elastase positive PMN is shown.

\section{Degradation of E-cadherin by PMN lysatel neutrophil elastase}

A potential target for neutrophil elastase on tumor cells is the adhesion molecule E-cadherin, which mediates the mutual binding. By indirect immunofluorescence and by flow cytometry, E-cadherin could be detected on SKOV-3 cells. Incubation of cells with either neutrophil elastase $(3 \mu \mathrm{g} / \mathrm{mL})$ or PMN lysate $(50 \mu \mathrm{L} / \mathrm{mL})$ for $5 \mathrm{~h}$ to $24 \mathrm{~h}$, caused a degradation of E-cadherin as seen by using an antibody that binds to the N-terminus of E-cadherin (Fig. 3). Flow cytometry showed a decrease of surface E-cadherin expression when cells were treated with elastase (on average $(n=3)$ reduction of mean fluorescence intensity of $70 \%)$. PMN lysates also reduced E-cadherin expres- sion (on average by 30\%) (Fig. 3A). Degradation of E-cadherin by PMN lysate or elastase was confirmed by Western blot analysis. Membrane expression was reduced, whereas cytosolic E-cadherin was not affected, being compatible with the notion that elastase targeted only the surface-expressed E-cadherin (one of three experiments is shown in Fig. 3B).

\section{Induction of an epithelial-to-mesenchymal transition (EMT) of SKOV-3 by elastase}

Whether dyshesion and shape changes of SKOV-3 are associated with an induction of EMT was evaluated in the next set of experiments. To this end, expression of cytokeratins as a marker for epithelial cells was assessed. By Western blotting, a decrease of cytokeratins was seen in cells treated with either 
PMN, PMN lysate, or elastase. Concomitantly, $\beta$-catenin expression increased in the nuclear fraction,

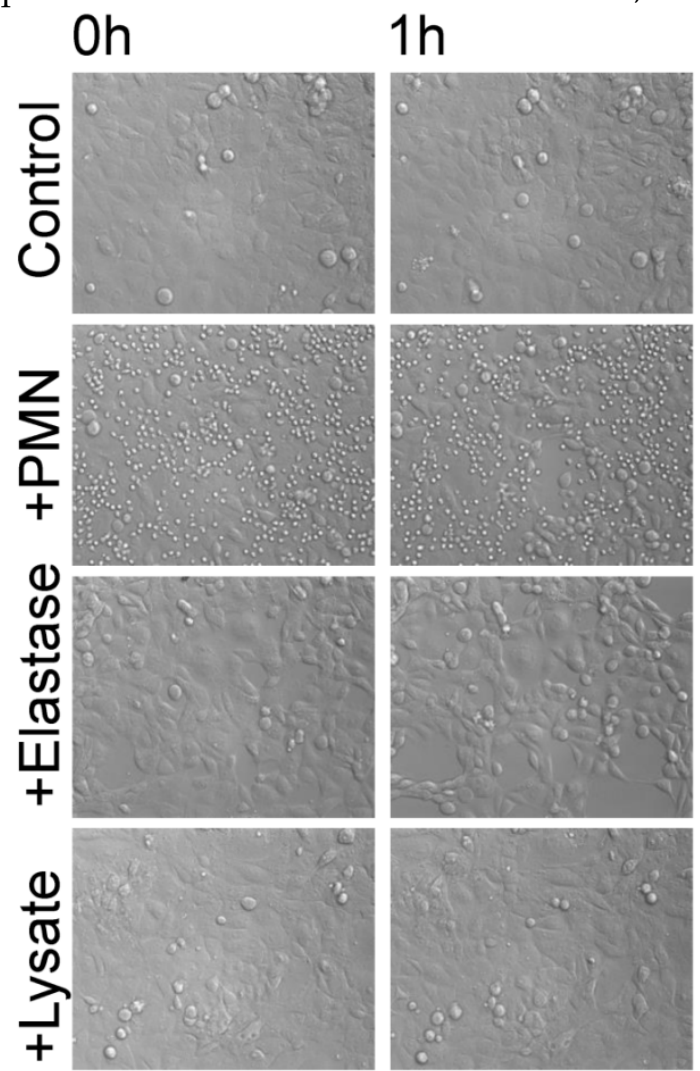

indicating a translocation of $\beta$-catenin to the nucleus as it occurs in the course of EMT (Fig. 4).

$3 h \quad 6 h$
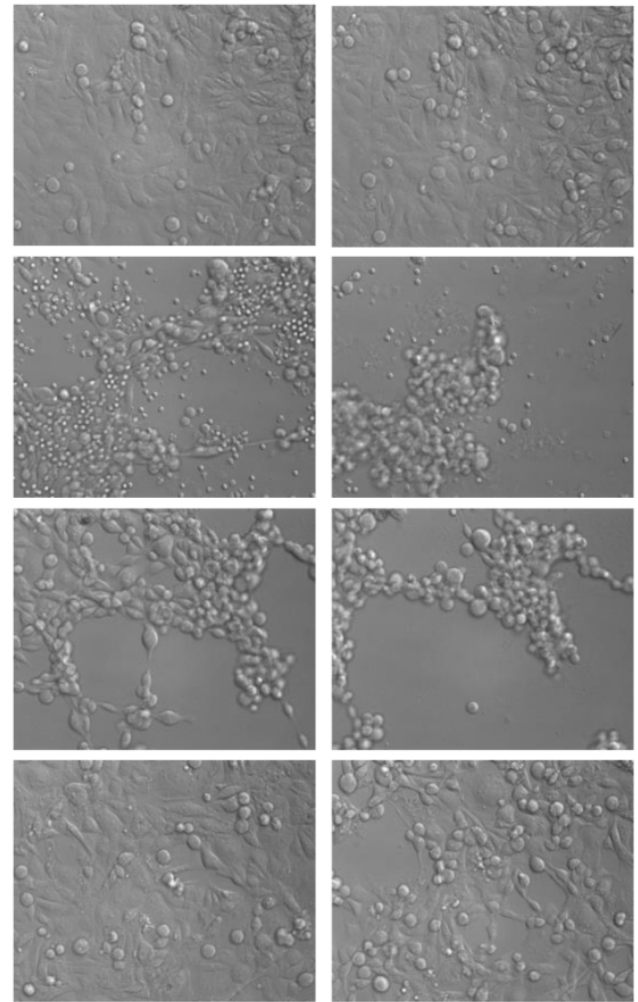

Figure 2. Effect of PMN on SKOV-3 monolayers: Tumor cells were grown to near confluence and PMN, PMN elastase, or PMN lysate were added and the interaction was observed by time-lapse video microscopy. Images were selected from the video clip after $0 \mathrm{~h}, 1 \mathrm{~h}, 3 \mathrm{~h}$, and $6 \mathrm{~h}$, respectively. The data are representative for three experiments performed with PMN derived from different individuals.

A

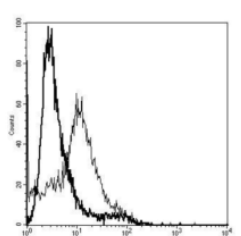

E-cadherin

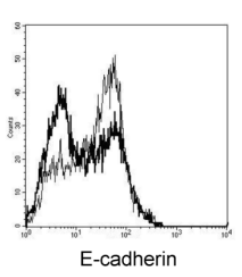

B cytoplasm membrane

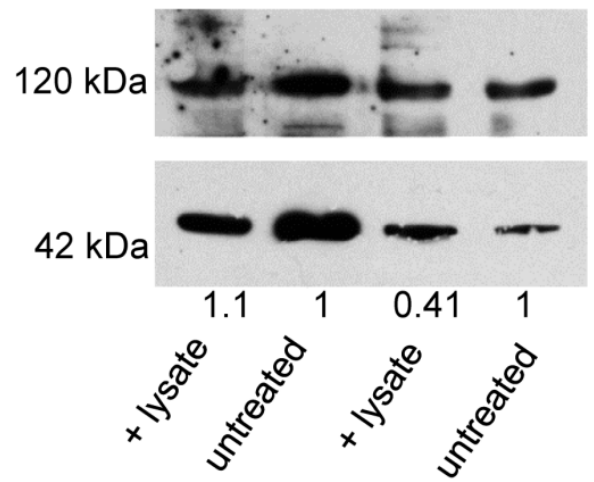

Figure 3. Effect of elastase or PMN lysate on E-cadherin surface expression: A By flow cytometry E-cadherin expression on SKOV-3 was determined on untreated cells (thin line) and after addition of elastase (left panel; thick lines) or PMN-lysate (right panel; thick line). Shown are representative examples. B Western blot analysis revealed a significant reduction of $\mathrm{E}$-cadherin in the cell membrane but not in the cytoplasm ( $\beta$-actin was used as loading control, relative densities were calculated with Quantity One Software (BioRad). Data shown are representative of three experiments.

\section{Functional consequences of EMT in SKOV-3}

To assess functions of SKOV-3 following EMT, mobility of cells was tested using an in vitro wound healing assay. As seen in the example in Fig. 4, following treatment with elastase, approximately 2.1 fold more cells migrated into the depleted area compared to untreated cells. On average, $26.7 \%$ of the depleted area was overgrown again compared to $12.7 \%$ in the control (mean of three experiments). 
A

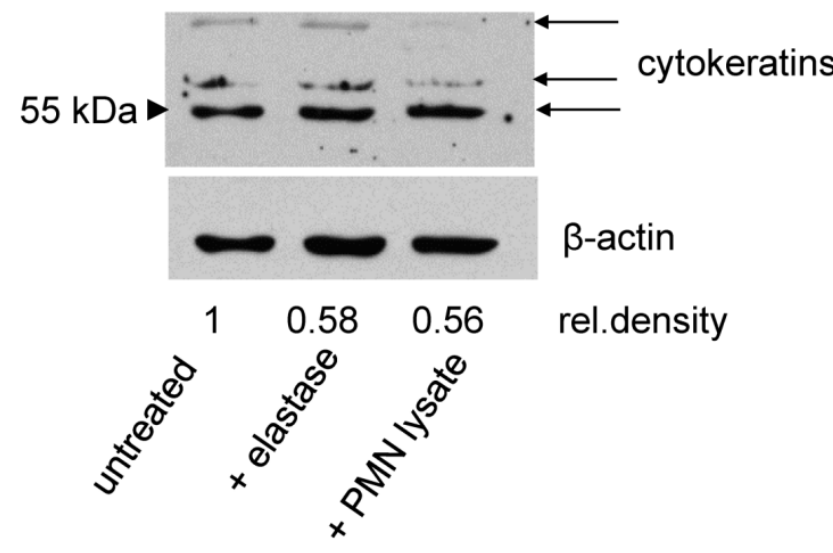

B nuclear fraction

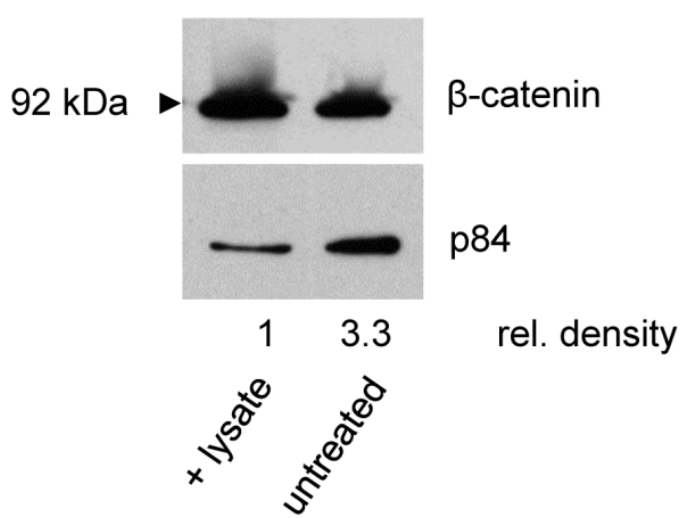

Figure 4. Induction of EMT in SKOV-3 by elastase or PMN lysate: A Cytokeratin (upper panel) was determined in the cell lysates of untreated SKOV-3 or of SKOV-3 being exposed to either PMN elastase or PMN lysate. The antibody recognized three cytokeratins, expression of which declined in treated cells. With $\beta$-actin as loading control, it was seen that the density of the cytokeratin bands declined in treated cells (the numbers indicate the relative density obtained for the $55 \mathrm{kD}$ band. Data of one of three experiments is shown). B In SKOV-3 treated with PMN lysate, a translocation into the nucleus of $\beta$-catenin was seen. As loading control the nuclear antigen p84 was used (one of three experiments is shown).

\section{Discussion}

The role of neutrophils in tumors is controversially discussed: on the one hand, induction of tumor cell apoptosis [17] or killing [18, 19] has been described, on the other hand an association with tumor progression, presumably due to promotion of metastasis [20], of angiogenesis or by dampening the anti-cancer immune reaction [21]. Moreover, neutrophil-derived elastase promotes tumor progression by increasing cell growth and survival [22]. Especially for ovarian cancers, it has been shown that (indirect) depletion of neutrophils in murine models causes disease stabilization, thus a pivotal role in ovarian cancer is likely [4].

In our collection with ovarian cancer samples, neutrophil infiltration into tumors was seen in the
A + PMN elastase untreated

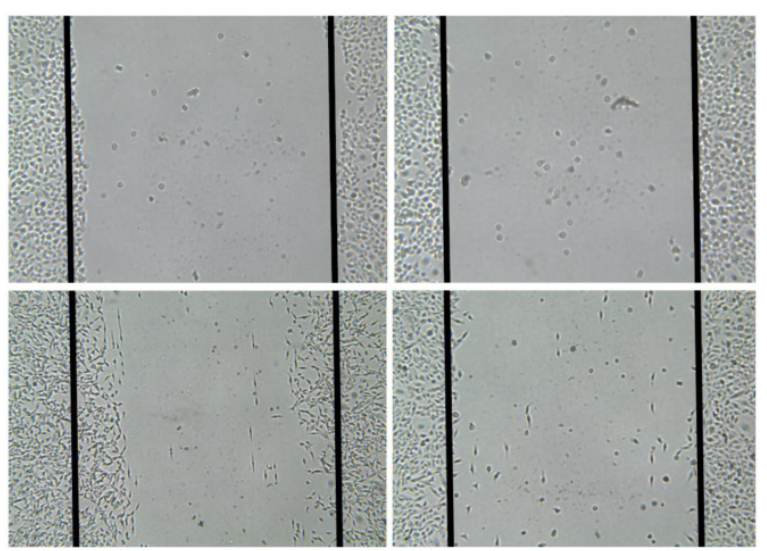

$\mathrm{Oh}$

B

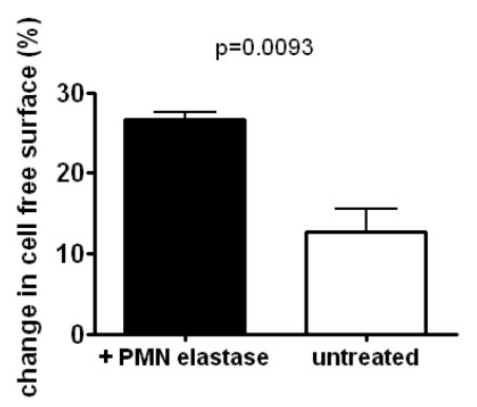

Figure 5. Migration of SKOV-3 cells: A Cells were grown to confluence. Then a defined area was scratched (time $0 \mathrm{~h}$ ) and the repopulation after 24h was determined by untreated cells or cells that had been pretreated with elastase. B Quantification of data of three experiments (mean + SD).

majority of cases. The infiltrated cells were not homogenously distributed; areas with dense PMN infiltrates were seen, but also those lacking infiltrating cells. Overall, the number of infiltrating neutrophils did not correlate with severity of disease or histologic classification. However, due to the inhomogeneous pattern, quantification has to be interpreted with some caution.

Epithelial cells and in that respect also ovarian tumor cells adhere to each other via E-cadherin. In our very large patient cohort E-cadherin expression was lowest in the most aggressive, high-grade serous subgroup as compared to the histological subtypes of the low-grade group. These results corroborate with findings that a loss of E-cadherin is associated with poor outcome in ovarian cancer patients [23]. In addition, a decreased E-cadherin expression was associ- 
ated with increased peritoneal metastasis and frequently seen in high-grade serous cancers [24]. Loss of surface-associated E-cadherin is - at least in part - due to cleavage by neutrophil-derived elastase, a fact that we confirmed in our vitro study. One have to keep in mind that a plethora of factors abundant in infiltrated cells of the tumor microenvironment, and in particular PMN, such as TGF- $\beta$ [25], amphiregulin [26], HIF-1a [27], or FGF-2 [28] are also able to induce E-cadherin downregulation in ovarian cancer. Loss of E-cadherin and the subsequent weakening of the cell-to-cell contacts by loss of cell polarity is considered to be a crucial step in the epithelial-to-mesenchymal transition process [29].

Elastase is one of the major effector proteins in neutrophils [30] linking the loss of E-cadherin to the infiltrating neutrophils. That E-cadherin is rapidly recycled from intracellular stores or de novo synthesized might explain the fact that there is no numerical correlation between E-cadherin expression and the leukocyte number. Of note, although elastase is the most likely effector in E-cadherin cleavage, other PMN-derived proteases cannot be ruled out.

Cleavage of surface-associated E-cadherin has numerous consequences; among those loss of cellular polarity and shape change; enhanced motility of the cells, and induction of epithelial-to-mesenchymal transition (EMT). Expression of ZEB1 in biopsies of patients is indicative of the latter, and although the overall number of ZEB1 positive cells did not correlate with the number of infiltrating cells, ZEB1 positive cells were accumulated in areas of dense neutrophil infiltrates.

A definite link between PMN and induction of EMT was established by our in vitro experiments. PMN and, more specifically, PMN-derived elastase cause a dyshesion of ovarian cancer cells grown in monolayers. Elastase cleaved surface E-cadherin of ovarian cancer cells and induced EMT, as determined by loss of cytokeratins and translocation into the nucleus of $\beta$-catenin. Moreover, an increased migratory potential of the ovarian cancer cells was observed. These findings are in line with data reported for pancreas epithelial cells in pancreatitis or pancreatic cancer cells [31], for lung cancer [32], colorectal cancer [33], or hepatocellular carcinoma [34]. The transition from an epithelial towards a mesenchymal phenotype is usually associated with a more aggressive phenotype of different tumors [35].

\section{Conclusion}

Human biopsies of a large tumor cohort showed neutrophil infiltrates as common feature in ovarian cancer. In the majority of cases, loss of E-cadherin on tumor cells was detected and EMT was seen. In vitro data established a link between neutrophil-derived elastase, loss of E-cadherin, and EMT, providing an explanation why ovarian cancer patients with an increased level on neutrophils have a poorer prognosis.

\section{Acknowledgements}

The authors thank Ms. Heike Conrad, Ms. Sarah Meßnard, Ms. Birgit Prior, and Mrs. Sabine Stegmaier for excellent technical support. The work of C.M. was funded by the Medical Faculty Heidelberg. The work of M.M.G. was funded by the German Research Foundation (SFB938).

\section{Authors' contributions}

C.M., A.S.M., K.H., M.M.G. planned and performed experiments. S.D.E, J.S. and I.B. performed the tissue microarray and collected clinical data. J.R. and C.S. discussed the data. C.M., G.M.H and M.M.G. analyzed the data and wrote the manuscript.

\section{Abbreviations}

EMT: epithelial-to-mesenchymal transition

FFPE: formalin-fixed and paraffin embedded

FIGO: international federation of gynecology and obstetrics

FITC: fluorescein isothiocyanate

H\&E: hematoxylin/eosin

OvCa: ovarian cancer

PMN: polymorphonuclear neutrophils

SDS-PAGE: sodium dodecyl sulfate polyacrylamide gel electrophoresis

SMA: smooth muscle actin

TOC: Tumorbank ovarian cancer

TRIS: Tris(hydroxymethyl)-aminomethan

ZEB1: Zinc Finger E-Box Binding Homeobox 1

\section{Competing Interests}

The authors have declared that no competing interest exists.

\section{References}

1. Siegel RL, Miller KD, Jemal A: Cancer statistics, 2015. CA Cancer J Clin 2015, 65:5-29.

2. McGuire WP, Brady MF, Ozols RF: The Gynecologic Oncology Group experience in ovarian cancer. Ann Oncol 1999, 10 Suppl 1·29-34.

3. Parmar MKB, Ledermann JA, Colombo N, du Bois A, Delaloye J-F, Kristensen GB, Wheeler S, Swart AM, Qian W, Torri V, Floriani I, Jayson G, Lamont A, Tropé C: Paclitaxel plus platinum-based chemotherapy versus conventional platinum-based chemotherapy in women with relapsed ovarian cancer: the ICON4/AGO-OVAR-2.2 trial. Lancet (London, England) 2003, 361:2099-106.

4. Balkwill FR, Capasso M, Hagemann T: The tumor microenvironment at a glance. J Cell Sci 2012, 125(Pt 23):5591-6.

5. Musrap N, Diamandis EP: Revisiting the complexity of the ovarian cancer microenvironment--clinical implications for treatment strategies. Mol Cancer Res 2012, 10:1254-64.

6. Lichtenstein A, Seelig M, Berek J, Zighelboim J: Human neutrophil-mediated lysis of ovarian cancer cells. Blood 1989, 74:805-9.

7. Williams KA, Labidi-Galy SI, Terry KL, Vitonis AF, Welch WR, Goodman A, Cramer DW: Prognostic significance and predictors of the neutrophil-to-lymphocyte ratio in ovarian cancer. Gynecol Oncol 2014, 132:542-50.

8. Cho KR: Ovarian cancer update: lessons from morphology, molecules, and mice. Arch Pathol Lab Med 2009, 133:1775-81. 
9. Klink M, Jastrzembska K, Nowak M, Bednarska K, Szpakowski M, Szyllo K, Sulowska Z: Ovarian cancer cells modulate human blood neutrophils response to activation in vitro. Scand J Immunol 2008, 68:328-36.

10. Aeed PA, Nakajima M, Welch DR: The role of polymorphonuclear leukocytes $(\mathrm{PMN})$ on the growth and metastatic potential of $13762 \mathrm{NF}$ mammary adenocarcinoma cells. Int J Cancer 1988, 42:748-59.

11. Welch DR, Schissel DJ, Howrey RP, Aeed PA: Tumor-elicited polymorphonuclear cells, in contrast to "normal" circulating polymorphonuclear cells, stimulate invasive and metastatic potentials of rat mammary adenocarcinoma cells. Proc Natl Acad Sci U S A 1989, 86:5859-63.

12. Marzano A V, Cugno M, Trevisan V, Lazzari R, Fanoni D, Berti E, Crosti C: Inflammatory cells, cytokines and matrix metalloproteinases in amicrobial pustulosis of the folds and other neutrophilic dermatoses. Int J Immunopathol Pharmacol , 2011;24:451-60.

13. Thiery JP, Sleeman JP: Complex networks orchestrate epithelial-mesenchymal transitions. Nat Rev Mol Cell Biol 2006, 7:131-42.

14. Jeanes A, Gottardi CI, Yap AS: Cadherins and cancer: how does cadherin dysfunction promote tumor progression? Oncogene 2008, 27:6920-9.

15. Rakha EA, Abd El Rehim D, Pinder SE, Lewis SA, Ellis IO: E-cadherin expression in invasive non-lobular carcinoma of the breast and its prognostic significance. Histopathology 2005, 46:685-93.

16. Baranwal S, Alahari SK: Molecular mechanisms controlling E-cadherin expression in breast cancer. Biochem Biophys Res Commun 2009, 384:6-11.

17. Stockmeyer B, Beyer T, Neuhuber W, Repp R, Kalden JR, Valerius T, Herrmann M: Polymorphonuclear granulocytes induce antibody-dependent apoptosis in human breast cancer cells. J Immunol 2003, 171:5124-9.

18. Otten MA, Rudolph E, Dechant M, Tuk CW, Reijmers RM, Beelen RHJ, van de Winkel JGJ, van Egmond M: Immature neutrophils mediate tumor cell killing via IgA but not IgG Fc receptors. J Immunol 2005, 174:5472-80.

19. Finisguerra V, Di Conza G, Di Matteo M, Serneels J, Costa S, Thompson AAR, Wauters E, Walmsley S, Prenen H, Granot Z, Casazza A, Mazzone M: MET is required for the recruitment of anti-tumoural neutrophils. Nature 2015, 522:349-53

20. Coffelt SB, Kersten K, Doornebal CW, Weiden I, Vrijland K, Hau C-S, Verstegen NJM, Ciampricotti M, Hawinkels LJAC, Jonkers J, de Visser KE: IL-17-producing $\gamma \delta \mathrm{T}$ cells and neutrophils conspire to promote breast cancer metastasis. Nature 2015, 522:345-8.

21. Fridlender ZG, Sun J, Kim S, Kapoor V, Cheng G, Ling L, Worthen GS, Albelda SM: Polarization of tumor-associated neutrophil phenotype by TGF-beta: "N1" versus "N2" TAN. Cancer Cell 2009, 16:183-94.

22. Houghton AM, Rzymkiewicz DM, Ji H, Gregory AD, Egea EE, Metz HE, Stolz DB, Land SR, Marconcini LA, Kliment CR, Jenkins KM, Beaulieu KA, Mouded M, Frank SJ, Wong KK, Shapiro SD: Neutrophil elastase-mediated degradation of IRS-1 accelerates lung tumor growth. Nat Med 2010, 16:219-23.

23. Faleiro-Rodrigues C, Macedo-Pinto I, Pereira D, Lopes CS: Prognostic value of E-cadherin immunoexpression in patients with primary ovarian carcinomas. Ann Oncol 2004, 15:1535-42.

24. Faleiro-Rodrigues C, Macedo-Pinto I, Pereira D, Ferreira VM, Lopes CS: Association of E-cadherin and beta-catenin immunoexpression with clinicopathologic features in primary ovarian carcinomas. Hum Pathol 2004, 35:663-9.

25. Oiu X, Cheng J-C, Klausen $C$, Fan $\mathrm{O}$, Chang H-M, So W-K, Leung PCK: Transforming growth factor- $\alpha$ induces human ovarian cancer cell invasion by down-regulating E-cadherin in a Snail-independent manner. Biochem Biophys Res Commun 2015, 461:128-35.

26. So W-K, Fan Q, Lau M-T, Qiu X, Cheng J-C, Leung PCK: Amphiregulin induces human ovarian cancer cell invasion by down-regulating E-cadherin expression. FEBS Lett 2014, 588:3998-4007.

27. Cheng J-C, Klausen C, Leung PCK: Hypoxia-inducible factor 1 alpha mediates epidermal growth factor-induced down-regulation of E-cadherin expression and cell invasion in human ovarian cancer cells. Cancer Lett 2013, 329:197-206.

28. Lau M-T, So W-K, Leung PCK: Fibroblast growth factor 2 induces E-cadherin down-regulation via PI3K/Akt/mTOR and MAPK/ERK signaling in ovarian cancer cells. PLoS One 2013, 8:e59083.

29. Kalluri R, Weinberg RA: The basics of epithelial-mesenchymal transition. I Clin Invest 2009, 119:1420-8.

30. Belaaouaj A, McCarthy R, Baumann M, Gao Z, Ley TJ, Abraham SN, Shapiro SD: Mice lacking neutrophil elastase reveal impaired host defense against gram negative bacterial sepsis. Nat Med 1998, 4:615-8.

31. Gaida MM, Steffen TG, Günther F, Tschaharganeh DF, Felix K, Bergmann F, Schirmacher P, Hänsch GM: Polymorphonuclear neutrophils promote dyshesion of tumor cells and elastase-mediated degradation of E-cadherin in pancreatic tumors. Eur J Immunol 2012, 42:3369-80.

32. Cui T, Srivastava AK, Han C, Yang L, Zhao R, Zou N, Qu M, Duan W, Zhang $X$, Wang Q-E: XPC inhibits NSCLC cell proliferation and migration by enhancing E-Cadherin expression. Oncotarget 2015, 6:10060-72.

33. Bi X, Pohl NM, Qian Z, Yang GR, Gou Y, Guzman G, Kajdacsy-Balla A, Iozzo $\mathrm{R} V$, Yang W: Decorin-mediated inhibition of colorectal cancer growth and migration is associated with E-cadherin in vitro and in mice. Carcinogenesis 2012, 33:326-30

34. Grosse-Steffen T, Giese T, Giese N, Longerich T, Schirmacher P, Hänsch GM, Gaida MM: Epithelial-to-mesenchymal transition in pancreatic ductal adenocarcinoma and pancreatic tumor cell lines: the role of neutrophils and neutrophil-derived elastase. Clin Dev Immunol 2012, 2012:720768.
35. Heerboth S, Housman G, Leary M, Longacre M, Byler S, Lapinska K, Willbanks A, Sarkar S: EMT and tumor metastasis. Clin Transl Med 2015, 4:6. 